\title{
Delayed allergy to acyclovir revealed by lymphocyte proliferation test
}

\author{
Marcello Albanesi ${ }^{1}$, Attilio Di Girolamo ${ }^{1}$, Vincenzo Aresta ${ }^{1}$, Maria Pia Rossi ${ }^{1}$, Lucia Giliberti ${ }^{1}$, Tommasina Perrone ${ }^{2}$, \\ Danilo Di Bona ${ }^{1}$, Maria Filomena Caiaffa ${ }^{3}$, Giorgina Specchia ${ }^{2}$, Luigi Macchia ${ }^{1}$
}

1Department of Emergency and Organ Transplantation, Section of Allergology and Clinical Immunology, University of Bari-Aldo Moro, Bari, Italy

2Department of Emergency and Organ Transplantation, Section of Haematology, University of Bari-Aldo Moro, Bari, Italy

${ }^{3}$ Department of Medical and Surgical Sciences, School and Chair of Allergology and Clinical Immunology, University of Foggia, Foggia, Italy

Adv Dermatol Allergol 2018; XXXV (5): 527-529

DOI: https://doi.org/10.5114/ada.2018.77244

Acyclovir (Acycloguanosine) is an antiviral drug commonly used in the management of the Herpes virus infections. Indeed, acyclovir has the unique property of inhibiting viral DNA polymerases and, thereby, decreasing the production of viral particles. Acyclovir can be administered by a topical, oral or intravenous route, depending on clinical circumstances. Importantly, in the case of haematological malignancies, acyclovir can be used after either chemotherapy or stem cell transplantation, in order to prevent Herpes virus reactivation [1]. So far, immediate allergic reactions to acyclovir have been described [2]. In contrast, delayed allergy to acyclovir remains anecdotal [3].

Here we report a case of an adult patient diagnosed with non-Hodgkin lymphoma who developed severe delayed hypersensitivity to oral acyclovir administration. The adverse reaction was revealed using a non-radioactive lymphocyte proliferation test (LPT).

A 62-year-old man was diagnosed with non-Hodgkin lymphoma in 2014. In May 2016, he underwent autologous haematopoietic stem cell transplantation, followed by an oral acyclovir treatment course $(1200 \mathrm{mg} /$ day, for 7 days), to prevent Herpes virus reactivation (IgG antiHerpes simplex 1-2 29.1 U/ml; IgG anti-Epstein Barr virus $30.4 \mathrm{U} / \mathrm{ml}$ ). This treatment was well tolerated.

In November 2016, due to a relapse of the non-Hodgkin lymphoma, the patient started treatment with bendamustine and rituximab. This treatment was followed by an oral acyclovir course at the same dosage as above. However, after 10 days, the patient developed a generalized cutaneous rash characterized by erythematous, long-lasting and intensively itchy lesions (Figure 1 A). For this reason, the treatment with acyclovir was stopped and treatment with prednisone $5 \mathrm{mg} /$ day and fexofenadine hydrochloride $180 \mathrm{mg}$ b.i.d. was established.
In December 2016, the patient underwent another treatment course with bendamustine and rituximab; however no antiviral treatment was administered following this latter treatment course. Importantly, the patient did not develop any adverse reactions.

Thereby, based on the clinical symptoms manifested, the time of onset of the adverse reaction and the resolution of the clinical condition upon treatment withdrawal and steroid treatment, we postulated the diagnosis of delayed hypersensitivity to acyclovir.

Thus, the case was further investigated using both patch tests and LPT. The patch tests performed with a $25 \mathrm{mg} / \mathrm{ml}$ solution of acyclovir were negative. As for the LPT, the lymphocytes of the patient were incubated for 5 days with 3 different 10-fold acyclovir concentrations: $3 \mu \mathrm{g} / \mathrm{ml}$, that is the "therapeutic concentration" calculated on a distribution volume of $9 \mathrm{l} / \mathrm{kg}$ [4], 0.3 and $30 \mathrm{\mu g} / \mathrm{ml}$, respectively. Upon incubation for $2 \mathrm{~h}$ with bromodeoxyuridine, lymphocyte proliferation was assessed using an anti-bromodeoxyuridine monoclonal antibody. The test is deemed positive when the proliferation rate of any of the three concentrations tested (compared to the control) equals or exceeds 2 [5]. The test provided a ratio of 2.41 for one of the three concentrations and was thereby considered positive (Figure $1 \mathrm{~B}$ ). To confirm this latter result we performed LPT with the acyclovir prodrug valacyclovir. Indeed, even though valacyclovir and acyclovir share the same core chemical structure, valacyclovir carries an L-valyl side chain. This different chemical structure could possibly modify the antigenicity of this drug compared to acyclovir (Figure $1 \mathrm{C}$ ). Thus, we used the same experimental settings described above and we tested 3 different 10-fold valacyclovir concentrations: $3.25,32.5$ (the therapeutic concentration)

Address for correspondence: Dr. Marcello Albanesi, Department of Emergency and Organ Transplantation, Section of Allergology and Clinical Immunology, University of Bari-Aldo Moro, Piazza Giulio Cesare, Policlinico, 70124 Bari, Italy, phone: +39 0805478817 , fax: +39080 5593576, e-mail: marcello.albanesi@uniba.it

Received: 9.05.2017, accepted: 23.08.2017. 
A

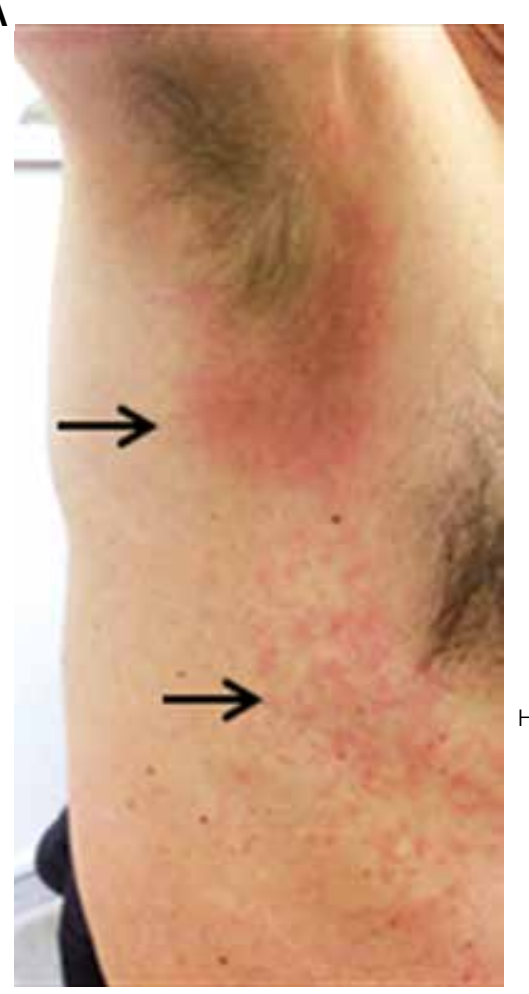

D

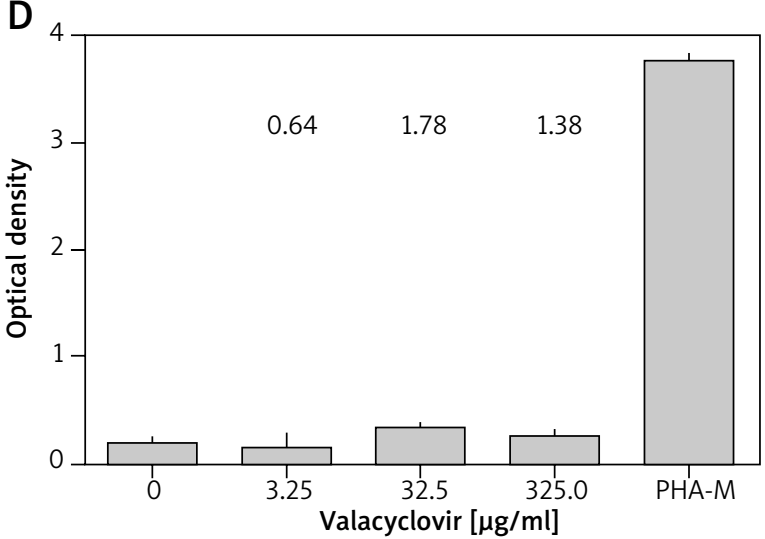

C<smiles>Nc1nc2c(ncn2COCCO)c(=O)[nH]1</smiles>

Acyclovir
B

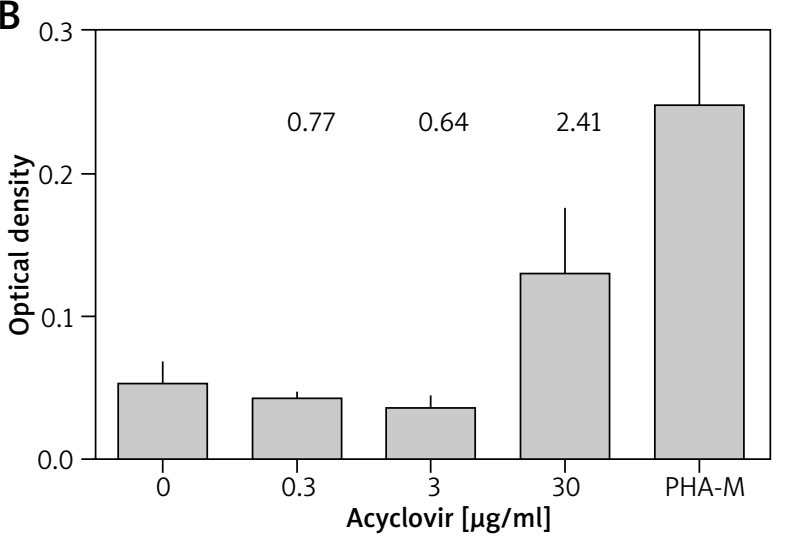<smiles>CC(C)C(N)C(=O)OCCOCn1cnc2c(=O)[nH]c(N)nc21</smiles>

Valacyclovir

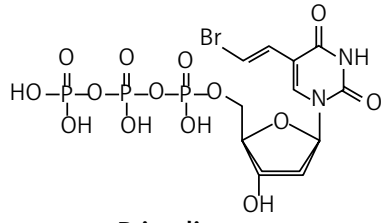

Brivudin

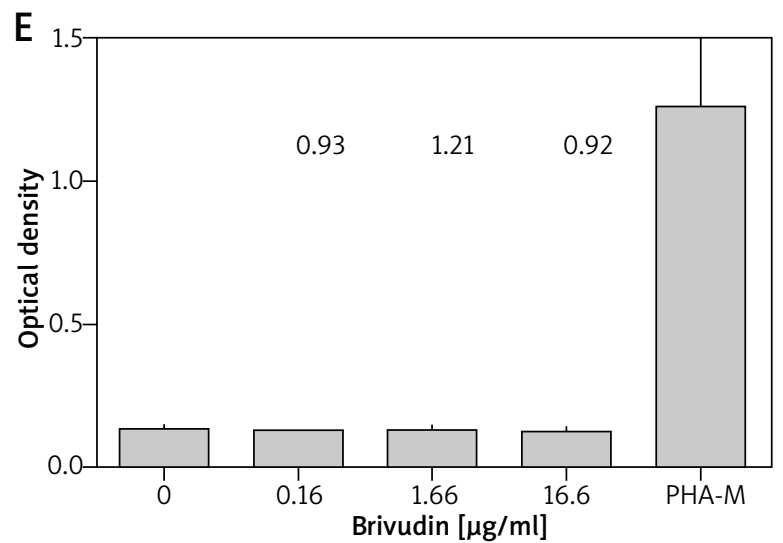

Figure 1. A-Representative picture of cutaneous lesions observed. Similar lesions were scattered on the whole body. B, D, E - lymphocyte proliferation assessed after 5 days of culture with the indicated concentrations of acyclovir (B), valacyclovir (D) and brivudin (E). Phytohaemagglutinin M (PHA-M) served as a positive control. The ratio between optical density relevant to the tested drugs (each at three 10-fold concentrations) and the negative control is shown above each histogram column. Error bars correspond to SEM. C - chemical structure of acyclovir (left panel), valacyclovir (middle panel) and brivudin (right panel)

and $325 \mu \mathrm{g} / \mathrm{ml}$, calculated on a distribution volume of $1.23 \mathrm{l} / \mathrm{kg}$ [4]. Interestingly, the LPT proved negative (Figure $1 \mathrm{D})$.

Moreover, in order to exclude the IgE-mediated nature of the adverse reaction, we performed skin tests with two distinct techniques: skin prick testing and intradermal testing. The patient was first subjected to skin prick testing, using a $25 \mathrm{mg} / \mathrm{ml}$ acyclovir solution and, successively, to intradermal tests with the offend- ing drug at 2 different 10-fold concentrations (viz. 0.25 and $2.5 \mathrm{mg} / \mathrm{ml}$ respectively). Both skin testing procedures proved negative.

Thus, taking into account the results of diagnostic tests performed, we confirmed the diagnosis of delayed allergy to acyclovir.

In order to allow future antiviral treatment (if needed), we tested brivudin as an alternative antiviral drug $[6,7]$. In order to ensure that brivudin was well tolerated, 
we performed both in vitro and in vivo tests. The LPT with 3 different 10 -fold concentrations of brivudin (viz. 0.16, 1.66 - the therapeutic concentration - and $16.6 \mu \mathrm{g} / \mathrm{ml}$, calculated on a distribution volume of 75 I) proved negative. Likewise, skin tests and patch tests proved negative. Finally, we performed an oral drug provocation test with brivudin (125 mg in 4 steps, 30 min apart) that was well tolerated. The drug was then administered for 3 consecutive days (125 mg, once daily) and no adverse reaction was observed.

To our knowledge, the clinical case presented in this report is the first acyclovir-induced delayed hypersensitivity demonstrated by LPT. It is well known that delayed hypersensitivity reactions involve $T$ cell activation. The offending drug can either activate $T$ cells by itself or via haptenization with proteins [8]. In the case of acyclovir, the exact mechanism by which $T$ cell activation is induced remains elusive. However, the positive LPT suggests that acyclovir might directly activate T cells. Indeed, it is unlikely that coupling with self-proteins might have occurred under in vitro LTP conditions.

In conclusion, the case reported shows that acyclovir can induce delayed hypersensitivity reactions. Moreover, aside from skin testing and patch testing techniques, LPT may be regarded as a useful diagnostic tool in the case of suspected delayed allergy to acyclovir.

Finally, as brivudin is structurally unrelated to acyclovir (Figure 1 C), it can be proposed as a suitable alternative antiviral drug in the case of delayed allergy to acyclovir.

\section{Conflict of interest}

The authors declare no conflict of interest.

\section{References}

1. Styczynski J, Reusser P, Einsele H, et al. Management of HSV, VZV and EBV infections in patients with hematological malignancies and after SCT: guidelines from the Second European Conference on Infections in Leukemia. Bone Marrow Transplant 2009; 43: 757-70.

2. Snape SE, Finch RG, Venkatesan P. Acyclovir desensitisation and rechallenge. BMJ Case Rep 2011; 2011: pii: bcr1020103392.

3. Robinson GE, Weber J, Griffiths C, et al. Cutaneous adverse reactions to acyclovir: case reports. Genitourin Med 1985; 61: 62-3.

4. Bomgaars L, Thompson P, Berg S, et al. Valacyclovir and acyclovir pharmacokinetics in immunocompromised children. Pediatr Blood Cancer 2008; 51: 504-8.

5. Porebski G, Gschwend-Zawodniak A, Pichler WJ. In vitro diagnosis of T-cell mediated drug allergy. Clin Exp Allergy 2011; 41: 461-70.

6. Meerbach A, Wutzler P, Gruhn B. Brivudin $\left(\right.$ Zostex $\left.^{R}\right)$ in the treatment of Herpes zoster in immunosoppressed patients. Antivir Res 2007; 74: A75.

7. Wutzler P, De Clercq E, Wutke K, Färber I. Oral brivudin vs. intravenous acyclovir in the treatment of herpes zoster in immunocompromised patients: a randomized double-blind trial. J Med Virol 1995; 46: 252-7.

8. Pichler WJ. The $p$-i concept: pharmacological interaction of drugs with immune receptors. World Allergy Organ J 2008; 1: 96-102. 